Geograficando, vol. 15, n. ${ }^{\circ}$ 1, e052, mayo-octubre 2019. ISSN 2346-898X

Universidad Nacional de La Plata.

Facultad de Humanidades y Ciencias de la Educación.

Departamento de Geografía

\title{
Mapeo de las políticas públicas vinculadas a la ESS en Uruguay (2015): Una perspectiva sobre su naturaleza y orientaciones a diez años de la asunción del gobierno progresista
}

\author{
Mapping of public policies related to the sse in uruguay (2015). A perspective on its nature and orientations ten years \\ after the assumption of the progressive government
}

\author{
Milton Torrelli \\ Unidad de Estudios Cooperativos, Servicio Central de Extensión y Actividades en el Medio, \\ Unidad de la República (UEC-SCEAM-UdelaR), \\ Uruguay \\ miltontorrelli@yahoo.com
}

Brisa De Giacomi

Integrante del Equipo Técnico del Proyecto Mapeo

Camila Falkin

Integrante del Equipo Técnico del Proyecto Mapeo

Gerardo Sarachu

Unidad de Estudios Cooperativos (UEC-SCEAM-UdelaR), Uruguay

\section{Resumen:}

Este trabajo mapea, caracteriza e identifica desafíos de las políticas públicas vinculadas al apoyo-fomento de la ESS en Uruguay. El proceso de identificación incluyó distintas fuentes, entre ellas el mecanismo de bola de nieve, y su relevamiento fue vía encuesta presencial. Si bien éstas crecieron durante los primeros diez años de gobierno progresista en el país (2005-2014), es necesario darle mayor densidad en cantidad, calidad y diversidad en la cobertura, así como enmarcarlas en una visión mayor y común que vaya más allá de la promoción de la ESS como herramienta de inclusión socio laboral. Finalmente, se reflexiona en torno a tres desafíos claves: profundizar el proceso de coproducción junto a otros actores de la ESS, mejorar su articulación interinstitucional y su transversalidad, y debatir-revisar la coherencia en la orientación conceptual y estratégica del conjunto de estas políticas en cuanto al por qué y el para qué apoyar-fomentar la ESS en el país.

Palabras clave: Participación, Articulación, Concepciones de ESS, Evaluación.

\begin{abstract}
:
This work maps, characterizes and identifies public policy challenges aimed to back the Social and Solidarity Economy (SSE) in Uruguay. The identification process included different sources, among them the snow ball technique, and the survey was done through interviews. These policies grew during the first ten years of the progressive government in the country (2005-2014). However, a stronger density in its quantity, quality and diversity as well as framing it in a broader and common vision than that of the SSE as a tool to foster labour and social inclusion is needed. Finally, the article reflects upon three key challenges: to deepen the co-production process with other actors of the SSE, to improve the inter institutional linkages and transversity, and to debate/ review the coherence in the conceptual and strategic orientation of these set of policies in terms of why and for what to support the SSE in Uruguay.
\end{abstract}

KEYWORDS: Participation, Articulation, SSE concepts, Evaluation. 


\section{A) INTRODUCCIÓN: FUNDAMENTACIÓN Y OBJETIVOS}

El estado -que se constituye por medio de las relaciones sociales, pero también las constituyeexpresa distintas correlaciones de fuerzas en cada momento histórico. Pero en tanto ninguna sociedad es homogénea, tampoco el estado es un bloque. En cada coyuntura, la hegemonía se construye y se disputa como resultado del carácter contradictorio de las relaciones sociales y del estado. Proponer actuar en y sobre el estado en la construcción de un proyecto alternativo reconoce esta dimensión contradictoria (de la forma y del aparato estado): 'luchar en y contra el

Estado, es, al mismo tiempo, luchar por clausurar sus instancias represivas y ampliar lo que tiene de socialidad colectiva' (Thwaites Rey, 2004: 80) (Hintze, 2010, p. 47).

En el siglo XXI, en el país y la región se han registrado cambios políticos que han impulsado un desarrollo significativo de las políticas hacia la Economía Social y Solidaria (ESS), ya sea directas o especializadas para la misma o bien una revalorización (las más de las veces instrumental) de lo asociativo y solidario para la resolución de necesidades.

En Uruguay, si bien existían algunos trabajos en la temática (Martí, Soria y Dabezies, 2008; Méndez y Romero, 2012; Confederación Uruguaya de Entidades Cooperativas -CUDECOOP-, 2014), se debía y debe profundizar y ser más sistemático en su relevamiento y análisis, al tiempo que a nivel regional surgían trabajos de análisis de tales políticas en perspectiva comparada que no abordaban el caso uruguayo (Hintze, 2010, Coraggio, 2014).

En ese contexto, este trabajo, en el marco de un proyecto mayor de "Mapeo, caracterización y desafíos de la Economía Solidaria (EcSol) en Uruguay", ${ }^{1}$ procuró relevar las políticas pública hacia la ESS (PPESS). Ello dado que se entiende que la EcSol o en general la ESS está compuesta por los emprendimientos de base y sus organizaciones/redes de articulación, pero que a ello están vinculadas entidades tanto públicas (las políticas aquí consideradas) como privadas de apoyo y fomento.

El gran objetivo del mapeo general fue justamente la búsqueda de saber qué características presentan y cuáles son los principales desafíos de cada uno de los actores que conforman el campo ESS en el país, en clave fundamentalmente de intercooperación, articulación y de construcción de movimiento. Es así que esas preocupaciones son el centro de las reflexiones finales con las que concluye este artículo.

Más allá de discusiones teóricas y estratégicas respecto a la importancia de la relación entre la ESS y el Estado, las políticas públicas estatales son un tema clave tanto para las posibilidades de sus emprendimientos como para la relevancia social y económica del sector/movimiento en su conjunto.

Ahora bien, es claro que se necesita para una ESS fuerte y de amplio alcance, no solo políticas exclusivas para ella, institucionalizadas en ciertos organismos y/o programas, sino que una de las demandas de la ESS es la interinstitucionalidad de las políticas, que en su conjunto deberían atender sus particularidades y promover su desarrollo, de cara a avanzar hacia "otra economía". Cuánto de políticas especializadas es necesario, es materia de debate. No obstante, ellas existen y tienen un importante impacto real y simbólico en el desarrollo de la ESS, de allí la relevancia de su relevamiento y análisis en este trabajo.

Siguiendo a Hintze (2010) se procura poner foco en ciertas características que deberían tener las políticas orientadas a la promoción y sostenibilidad de la ESS:

a) políticas en sentido amplio, no solo sociales,

b) interinstitucionales, dado que no pueden ser solo objeto de ciertos órganos gubernamentales,

c) interjuridiccionales, las experiencias de ESS están ancladas en procesos de desarrollo local más amplios, deben ser objeto de los distintos niveles del Estado (nacional, municipal),

d) participativas, bajo un proceso horizontal y dinámico que involucra diversos actores, vía diálogo y aprendizaje continuo.

En este punto, en Chaves (2012, p. 178) se reafirma la trascendencia para el sector de la participación en la coconstrucción de políticas públicas, y no solo en las vinculadas directamente a la ESS, al tiempo que señala una serie de ventajas para los poderes públicos de dicha participación. 
Por un lado, con la integración del sector, se profundiza en la democratización del proceso político, dado que sus entidades tienen estructuras de participación más democráticas que otros grupos de presión, al tiempo incluyen sectores sociales en general insuficientemente representados en los procesos políticos. Por otro, se mejoraría la eficacia de las políticas, en tanto la cercanía con la sociedad civil y sus intereses y necesidades de las entidades del sector, mejorarían la elección de objetivos e instrumentos en la fase de diseño y favorecerían el grado de aceptación de las mismas y con ello la eficacia en su aplicación.

En este trabajo sumamos a esos ejes el tratamiento del punto de partida conceptual desde el que trabajan cada una de las políticas, entendiendo que ello ayuda a comprender algunas de sus particularidades así como se torna un desafío para el conjunto de las mismas respecto a por qué y para qué fomentar los emprendimientos de ESS o sus organizaciones/redes en el país; lo que lógicamente implica el debate de cómo se lo hace.

\section{B) Aspectos conceptuales y metodológicos del Mapeo de las PPESS}

El mapeo general se centró en la ESS de naturaleza (primordialmente) económica, es decir, emprendimientos de esta naturaleza y sus organizaciones/redes, y por tanto, entidades de apoyo público y privadas a ellos dirigidas; siendo este un primer nivel de recorte de las políticas consideradas.

Asimismo, debe sealarse que en dicho mapeo se incluyeron tanto emprendimientos asociativos como familiares e individuales vinculados voluntariamente a redes de ESS, los que según ciertas formulaciones teóricas de la ESS no formarías parte de la misma. ${ }^{2}$

Se partió de una definición de PPESS que incluye a los proyectos, programas o planes de acción que son desarrollados por órganos de administración de los distintos niveles estatales con el objetivo de apoyo y/o fomento directo o indirecto a ESS. Las directas son las especializadas en la ESS, creadas para ella, las indirectas aquellas que incluyen a la ESS entre sus objetivos o herramientas, conjuntamente con otros agentes u objetivos involucrados en la política.

Dicha clasificación devino luego en tres categorías, según el apoyo a los emprendimientos de ESS o a sus organizaciones/redes de articulación sea:

1. el fundamento central de la política (alto nivel de vínculo en el apoyo a la ESS),

2. una herramienta priorizada para el logro de los objetivos de la política (media vinculación),

3. un ámbito más de la política como un todo (baja vinculación).

El trabajo de identificación (mapeo) de dichas políticas implicó cuatro tipos de fuentes:

i. trabajos previos (Méndez y Romero, 2012; Torrelli, 2013; CUDECOOP, 2014),

ii. búsqueda y lectura de materiales en sitios web de organismos públicos,

iii. entrevistas a actores calificados, $y$

iv. encuesta a los responsables de las políticas, y a partir de ello la visualización de otras políticas vía mecanismo de 'bola de nieve' (si bien como se verá luego, el conocimiento de otras políticas vinculadas no es muy elevado). ${ }^{3}$

Así se confeccionó el listado. Luego, en campo el criterio eliminatorio fue la pregunta: ¿la política busca apoyar en particular emprendimientos asociativos o de ESS en general o de sus organizaciones/redes de articulación? Si contestaba que no, se acababa la encuesta.

Vale señalar que la técnica de encuesta tiene limitaciones de alcance y profundidad naturales, pero fue la utilizada en el mapeo general dado el muy alto número de casos entre los distintos actores relevados (emprendimientos, organizaciones, redes-gremiales y pp).

El pretesteo se realizó a fines de 2014 y el relevamiento en el primer semestre de 2015.

Se identificaron 23 PPESS, vinculadas a 11 instituciones públicas distintas (Cuadro 1): Ministerio de Desarrollo Social (MIDES), Ministerio de Industria, Energía y Minería (MIEM), Ministerio de Ganadería, Agricultura y Pesca (MGAP), Ministerio de Trabajo y Seguridad Social (MTSS), Ministerio de 
Vivienda, Ordenamiento Territorial y Medio Ambiente (MVOTMA), Presidencia-Oficina de Planificación y Presupuesto (OPP), Intendencia de Montevideo (IM), Intendencia de Canelones (IC), Intendencia de Artigas (IA), Instituto Nacional de Colonización (INC) e Instituto Nacional del Cooperativismo (INACOOP). ${ }^{4}$

Si bien se identificaron algunas políticas más, los análisis de las mismas mostraron que, al ampliar, por ejemplo, a institutos públicos de derecho privado dedicados a ciertas temáticas específicas o a las oficinas de desarrollo de las intendencias, el apoyo o tratamiento de la ESS resultaba solo marginal, no pudiéndose incluir en este mapeo. Asimismo, a medida que se incursiona en políticas vinculadas a las MIPYMES en general, ocurre algo similar.

\section{CUADRO 1}

Identificación, alcances y objetivos de las políticas relevadas

\begin{tabular}{|c|c|c|c|c|c|c|c|c|c|}
\hline \multirow{2}{*}{\multicolumn{2}{|c|}{ Organismo }} & \multirow[b]{2}{*}{ Politica } & \multicolumn{2}{|c|}{ Alcance } & \multicolumn{3}{|c|}{ Ámbito principal } & \multirow{2}{*}{\multicolumn{2}{|c|}{ Principales dos objetivos de la poltica }} \\
\hline & & & 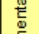 & $\overline{\mathrm{g}}$ & & & & & \\
\hline \multirow{6}{*}{\multicolumn{2}{|c|}{ MIDES }} & Uruguay dasifica & & $x$ & & $x$ & & $\begin{array}{l}\text { Contribuir al desarrollo de los proceso sintegrales para } \\
\text { la inclusión social de los dasificadores }\end{array}$ & \\
\hline & & Apoyo a emprendimientos & & $x$ & & & $x$ & $\begin{array}{l}\text { Fortalecer las estrate gias de generación de inte gración } \\
\text { productiva }\end{array}$ & $\begin{array}{l}\text { Contribuir a mejorar las condiciones de vida de los } \\
\text { emprendimientos }\end{array}$ \\
\hline & & $\begin{array}{l}\text { Marca social (sub programa PROVAS- } \\
\text { producto con va lor social) }\end{array}$ & & $x$ & & $x$ & & $\begin{array}{l}\text { Promover y forta lecer la participación de } \\
\text { emprendimiento sy a cores sociales relacion ados }\end{array}$ & $\begin{array}{l}\text { Comercialización local y regional. Promovery } \\
\text { apoyar estrategias innovadoras en el tema. }\end{array}$ \\
\hline & & $\begin{array}{l}\text { Programa de fortalecimiento de } \\
\text { emprendimientos productivos }\end{array}$ & & $x$ & & $x$ & & $\begin{array}{l}\text { Contribuir a la integración productiva autónoma de } \\
\text { personas y emprendimientos }\end{array}$ & $\begin{array}{l}\text { Para mejorar la producción, comercialización y } \\
\text { gestión }\end{array}$ \\
\hline & & Estrategia de ruralidad & & $x$ & $x$ & & & $\begin{array}{l}\text { Acceso a bienes, servicios y presta ciones de la } \\
\text { población rural }\end{array}$ & $\begin{array}{l}\text { Fortalecimiento de redes locale sy a sociativismo. } \\
\text { ortale cimiento de cap acidades }\end{array}$ \\
\hline & & Cooperativas sociales & & $x$ & & $x$ & & $\begin{array}{l}\text { Otorgar a las personas un instrumento legal de } \\
\text { formalización laboral }\end{array}$ & $\begin{array}{l}\text { Recuperación de los derechos ciudadan os de las } \\
\text { personas }\end{array}$ \\
\hline \multirow{3}{*}{\multicolumn{2}{|c|}{ MIEM }} & Desarrollo local & & $x$ & & $x$ & & Fortalecimiento de capacidades locales & Promoción del entorno de lasMIPYMES \\
\hline & & Promoción de desarrollo de artesanías & & $\mathrm{x}$ & & $\mathrm{x}$ & & $\begin{array}{l}\text { Desarrollar la artesanía como sustento de los } \\
\text { arte sanos }\end{array}$ & Construir un artesanal de calidad \\
\hline & & $\begin{array}{l}\text { Fortalecimiento e implementación de } \\
\text { polticicas de especialización productiva }\end{array}$ & & $x$ & & & $x$ & Fortalecimiento de emprendimientos productivos & Internacionalización \\
\hline \multirow{3}{*}{\multicolumn{2}{|c|}{ MGAP }} & $\begin{array}{l}\text { Fortalecimiento institucional } \\
\text { organizacional - M ontevideo rural }\end{array}$ & & $x$ & $x$ & & & $\begin{array}{l}\text { Generar capacidades en varios niveles, } \\
\text { multidimensionales en zonas rurales de fomento }\end{array}$ & $\begin{array}{l}\text { Que se conviertan en un canal de polticas } \\
\text { públicas }\end{array}$ \\
\hline & & Pro grama de micro cré dito rural & & $x$ & $x$ & & & $\begin{array}{l}\text { Acceso a crédito de la población rural al que no } \\
\text { accedian }\end{array}$ & $\begin{array}{l}\text { Generar organizaciones lo cales / comunitarias a } \\
\text { través de los comités de crédito }\end{array}$ \\
\hline & & Fondo rotato rio & & $x$ & $x$ & & & $\begin{array}{l}\text { Generar financiamiento a propuestas productivas de } \\
\text { producores rurales }\end{array}$ & Fortalecer la organización me diante herramientas \\
\hline \multicolumn{2}{|r|}{ MTSS } & Programa de inversión productiva & & $\mathrm{x}$ & & & $x$ & $\begin{array}{l}\text { Fomento y apoyo a unid ades productivas de pequeño } \\
\text { y mediano porte }\end{array}$ & \\
\hline \multicolumn{2}{|r|}{ MVOTMA } & Cooperativas de vivienda & & $x$ & & $x$ & & $\begin{array}{l}\text { Proveer viviendas ade cuadas y dignas a los a sociados } \\
\text { de las cooperativas }\end{array}$ & $\begin{array}{l}\text { Promover el cooperativismo por su forma de } \\
\text { organización democrática }\end{array}$ \\
\hline \multirow{2}{*}{ 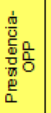 } & \multirow{2}{*}{ OPP } & Fondes (unidad técnica) & & $x$ & & & $x$ & Generar emprendimientos sólidos y autogestionarios & $\begin{array}{l}\text { Generar proyectos sustentables y brindar } \\
\text { asesoramiento té cnico }\end{array}$ \\
\hline & & $\begin{array}{l}\text { Uruguay más cerca (desarrollo económico } \\
\text { local) }\end{array}$ & & $\mathrm{x}$ & & & $\mathrm{x}$ & Disminución de los desequilibrios a nivel territorial & $\begin{array}{l}\text { Favorecer procesos de desarrollo económico a } \\
\text { nivel teritorial }\end{array}$ \\
\hline \multirow{5}{*}{ 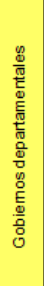 } & \multirow{2}{*}{ Montevideo } & Proyecto de desarrollo local & $x$ & & & & $x$ & $\begin{array}{l}\text { Indusión social de la comunidad a tavés del } \\
\text { desarrollo económico... }\end{array}$ & \\
\hline & & Estructura de producción familiar & $x$ & & $x$ & & & Desarrollo y mantenimien to del sistema produ ctivo & $\begin{array}{l}\text { Desarollo de siste mas productivos que respeten el } \\
\text { medio ambiente }\end{array}$ \\
\hline & \multirow{2}{*}{ Canelones } & Mipymes & $x$ & & & & $x$ & Fortalecimiento de las MIPYMES & Capacitación y microfinanciamiento \\
\hline & & Apoyo a la Economía Social & $\mathrm{x}$ & & & & $x$ & Indusión con tra bajo & Capacitación y educación \\
\hline & Artigas & $\begin{array}{l}\begin{array}{l}\text { Paeyc- Programa Artigas emprendedory } \\
\text { cooperativo }\end{array} \\
\end{array}$ & $x$ & & & & $x$ & Fomento del emprendedurismo & $\begin{array}{l}\text { Instrumenta una alte mativa para mejorar el nivel } \\
\text { de ocupa ción }\end{array}$ \\
\hline \multirow{2}{*}{ 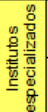 } & & Adjudicación asociativa de tierras & & $\mathrm{x}$ & $\mathrm{x}$ & & & El desarrollo rural & $\begin{array}{l}\text { El bienestar del tra bajador rural (asalariad oy } \\
\text { productor) }\end{array}$ \\
\hline & INACOOP & INACOOP & & $x$ & & $x$ & & $\begin{array}{l}\text { Programas de brmación, estudios e investigación, } \\
\text { promoción de procesos asociativos }\end{array}$ & Formulación de planes de desarrollo cooperativo \\
\hline
\end{tabular}


No obstante, hubo una ampliación del campo que resulta relevante frente a los trabajos existentes previamente, lo que podrá continuarse en el futuro con nuevas investigaciones. En todo caso, están todas las PPESS directas existentes y más allá de que puede sobrar alguna o faltar algunas otras en el total, se entiende que en líneas generales se describe en buena forma el panorama del conjunto de políticas vinculadas a la ESS en Uruguay.

Otro problema consistió en el nivel seleccionado de corte para definir planes de acción (políticas propiamente dicho, si bien aquí se utiliza políticas en genrico), proyectos, o programas. Es decir, en ocasiones una política representa un caso del mapeo, aunque est compuesta por distintos programas. En otras situaciones, programas aislados corresponden también a un caso. El punto de corte procura mantener la 'unidad lógica' de las políticas. ${ }^{5}$

A su vez, existen políticas con componentes vinculados a la ESS y otros que refieren a otras temáticas. En esos casos, se generó cierto grado de ambigüedad, ya que algunas respuestas refieren al conjunto de la política y otras se focalizan en sus aspectos vinculados a la ESS; lo que se intentó matizar realizando diferentes preguntas de 'control' y específicas.

Como se verá, el resultado ofrece una diversidad de políticas en muchos aspectos, pero claramente en materia de tamaño de los recursos involucrados, incluyendo desde pequeños proyectos dentro de ministerios o intendencias, hasta políticas de fuerte alcance e impacto como la política hacia las cooperativas de vivienda del MVOTMA.

$\mathrm{Al}$ respecto, vale sealar que salvo excepciones (INACOOP, MVOTMA e INC) se trata de recursos que no llegan a 5\% del presupuesto del organismo de pertenencia, sea ministerios, intendencias o PresidenciaOPP, siendo incluso en más del $50 \%$ menores a $1 \%{ }^{6}$

La diversidad es parte de lo que se quiere observar en un mapeo que procura relevar el conjunto, para tener una idea general sobre dónde y cómo se presenta la ESS en el país.

\section{c) Resultados y discusión}

\section{C.1. Caracterización general}

Solo 35\% de las PPESS relevadas tiene el apoyo a emprendimientos de ESS o a sus redes como fundamento de la política ( 8 casos), $40 \%$ lo considera una herramienta priorizada ( 9 casos), y el restante $25 \%$ lo considera un ámbito más de la política ( 6 casos). Es decir que existen 8 casos de apoyo directo y 15 de apoyo indirecto (vínculo medio o bajo) (Cuadro 2).

$\mathrm{Al}$ respecto existe un claro vínculo entre la existencia de un fondo especialmente destinado al asociativismo o la ESS en general (65\% de los casos, mientras que en las restantes 8 políticas el apoyo a la ESS no está diferenciado) y el nivel de vínculo con el apoyo a la ESS. Prácticamente todas las políticas de alto vínculo poseen a su vez un fondo específico y este es del $100 \%$ de los recursos disponibles, mientras que en el otro extremo, solo $50 \%$ de las políticas con bajo vínculo lo tiene y en ninguna implica el total de los recursos.

Como surge del Cuadro 2, de las 23 políticas solo 6 son previas a 2005. Las más viejas, de los noventa, más allá de que, salvo la de vivienda - muy concreta en su especificidad-, son de baja vinculación a la ESS. Una de ellas impulsada por el primer gobierno del Frente Amplio (FA) en el país al mando de la IM en 1990. Luego en 2003-2004 surgieron 2 vinculadas a fondos (uno rotatorio para emprendimientos y otro de microcréditos) para encarar el duro momento que presentaba la producción rural luego de la crisis de 2001-2002. 
CUADRO 2

Descripción general de las políticas relevadas

\begin{tabular}{|c|c|c|c|c|c|c|c|c|c|c|c|}
\hline \multirow{2}{*}{\multicolumn{2}{|c|}{ Organismo }} & \multirow[b]{2}{*}{ Nombre de la Politica } & \multirow[b]{2}{*}{$\begin{array}{l}\frac{0}{0} \\
\frac{0}{5} \\
\frac{0}{\circ} \\
\frac{0}{4}\end{array}$} & \multicolumn{3}{|c|}{ Apoyoa ESS } & \multirow[b]{2}{*}{ 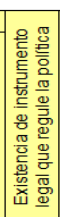 } & \multirow[b]{2}{*}{$\begin{array}{l}\text { Principal área de } \\
\text { acción }\end{array}$} & \multicolumn{3}{|c|}{ Principal fin } \\
\hline & & & & 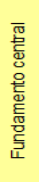 & 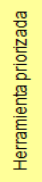 & 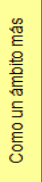 & & & 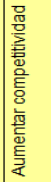 & 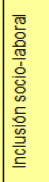 & 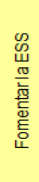 \\
\hline \multirow{6}{*}{\multicolumn{2}{|c|}{ MIDES }} & U ruguay clasifica & 2006 & $\mathrm{x}$ & & & $x$ & Trabajo y Empleo & & $x$ & \\
\hline & & Apoyo a emprendimientos & 2006 & & & $x$ & $x$ & Trabajo y Empleo & & $\mathrm{x}$ & \\
\hline & & $\begin{array}{l}\text { Marca social (sub programa PROVAS - } \\
\text { producto con valor social) }\end{array}$ & 2014 & & $x$ & & & Trabajo y Empleo & $x$ & & \\
\hline & & $\begin{array}{l}\text { Programa de fortalecimiento de } \\
\text { emprendimientos productivos }\end{array}$ & 2006 & & $x$ & & & Trabajo y Empleo & & $x$ & \\
\hline & & Estrategia de ruralidad & 2013 & & $\mathrm{x}$ & & $\mathrm{x}$ & \begin{tabular}{|l|} 
Bienestar y \\
Desarrollo Social
\end{tabular} & & $\mathrm{x}$ & \\
\hline & & Cooperativas sociales & 2006 & $\mathrm{x}$ & & & $x$ & Trabajo y Empleo & & $\mathrm{x}$ & \\
\hline \multirow{3}{*}{\multicolumn{2}{|c|}{ MIEM }} & Desarrollo local & 2010 & & & $x$ & & $\begin{array}{l}\text { Desarrollo } \\
\text { ec onomico local }\end{array}$ & $x$ & & \\
\hline & & Promoción de desarrollo de artesanfas & 1991 & & & $x$ & $x$ & $\begin{array}{l}\text { otra: } \\
\text { comercialización }\end{array}$ & $x$ & & \\
\hline & & $\begin{array}{l}\text { Fortalecimiento e implementación de } \\
\text { politicas de especialización productiva }\end{array}$ & 2009 & & $x$ & & $x$ & $\begin{array}{l}\text { Otra: desarrollo } \\
\text { productivo }\end{array}$ & $x$ & & \\
\hline \multirow{3}{*}{\multicolumn{2}{|c|}{ MGAP }} & $\begin{array}{l}\text { Fortalecimiento institucional } \\
\text { organizacional - Montevideo rural }\end{array}$ & 2007 & $x$ & & & $x$ & $\begin{array}{l}\text { Educación y } \\
\text { Capacitación }\end{array}$ & & & $\mathrm{x}$ \\
\hline & & Programa de mic rocrédito rural & 2004 & & $\mathrm{x}$ & & & $\begin{array}{l}\text { Desarrollo } \\
\text { económico local }\end{array}$ & & & $x$ \\
\hline & & Fondo rotatorio & 2003 & $\mathrm{x}$ & & & $x$ & $\begin{array}{l}\text { Desamollo } \\
\text { agropec uario }\end{array}$ & $\mathrm{x}$ & & \\
\hline \multicolumn{2}{|r|}{ MTSS } & Programa de inversión productiva & 1996 & & $x$ & & $x$ & Trabajo y Empleo & & $x$ & \\
\hline \multicolumn{2}{|c|}{ MVOTMA } & Cooperativas de vivienda & 1994 & $\mathrm{x}$ & & & $x$ & Otra: vivienda & & $x$ & \\
\hline \multirow{2}{*}{ 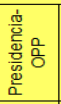 } & \multirow{2}{*}{ OPP } & Fondes (unidad técnica) & 2012 & $\mathrm{x}$ & & & $\mathrm{x}$ & Trabajo y Empleo & & & $x$ \\
\hline & & $\begin{array}{l}\text { Uruguay más cerca (desarrollo } \\
\text { económico local) }\end{array}$ & 2014 & & & $\mathrm{x}$ & & \begin{tabular}{|l|} 
Desarrollo \\
ec onómico local
\end{tabular} & $\mathrm{x}$ & & \\
\hline \multirow{5}{*}{ 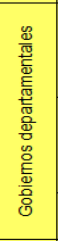 } & \multirow{2}{*}{ Montevideo } & Proyecto de desarrollo local & 2007 & & $\mathrm{x}$ & & $\mathrm{x}$ & $\begin{array}{l}\text { Desarrollo } \\
\text { ec onómico local }\end{array}$ & & $x$ & \\
\hline & & Estructura de producción familiar & 1990 & & $x$ & & $\mathrm{x}$ & 98 & & & $\mathrm{x}$ \\
\hline & \multirow{2}{*}{ Canelones } & Mipymes & 2010 & & & $\mathrm{x}$ & $\mathrm{x}$ & \begin{tabular}{|l|} 
Desamollo \\
ec onómico local
\end{tabular} & $\mathrm{x}$ & & \\
\hline & & Apoyo a la Economfa Social & 2005 & $x$ & & & & Trabajo y Empleo & $x$ & & \\
\hline & Artigas & $\begin{array}{l}\text { Paeyc - Programa Artigas emprendedor y } \\
\text { cooperativo }\end{array}$ & 2012 & & & $x$ & $x$ & Trabajo y Empleo & & $x$ & \\
\hline \multirow{2}{*}{ 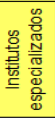 } & INC & Adjudicación asociativa de tierras & 2005 & & $\mathrm{x}$ & & $\mathrm{x}$ & Trabajo y Empleo & & $\mathrm{x}$ & \\
\hline & INACOOP & INACOOP & 2010 & $\mathrm{x}$ & & & $\mathrm{x}$ & $\begin{array}{l}\text { Educación y } \\
\text { Capacitación }\end{array}$ & & $\mathrm{x}$ & \\
\hline
\end{tabular}

Fuente: elaboración propia

En su gran mayoría, las políticas relevadas son resultado de la gestión de los dos primeros gobiernos del FA 2005-2009 y 2010-2014 (75\%). Nueve (40\%) se generaron en el primer gobierno del Frente Amplio (FA) bajo la presidencia de Vázquez, y las restantes ocho (35\%) en su segundo gobierno bajo la presidencia de Mujica, si bien en algún caso venían previstas desde el período anterior, como sucede con la creación del INACOOP que se consolida en 2010. Vale destacar que las 5 políticas implementadas por intendencias, en todos los casos corresponden a gobiernos del FA en ellas.

El primer gobierno del FA parece haber sido el más fructífero en este tipo de políticas, no solo por su cantidad, sino por su nivel de vínculo con la temática: 4 de vínculo alto, 4 medio y 1 bajo. Su segundo gobierno, presenta, inversamente, 2-2-4, si bien este gobierno de Mujica se caracterizó por impulsar ciertas políticas de fuerte compromiso con la ESS.

Vale señalar aquí que una de las debilidades para el análisis temporal que presenta este relevamiento es la imposibilidad de visualizar la profundización de políticas previas que puede darse durante un gobierno. Solo a modo de ejemplo, las cooperativas de vivienda existen desde hace cinco décadas -si bien la política aquí relevada plantea un inicio en 1994-, pero es indudable que su fomento se ha multiplicado durante la última década.

Por organismos públicos, se observa lo activo del uso de la ESS desde su aporte al desarrollo social y combate a la pobreza y exclusión, por otro, es destacable la presencia de políticas (aunque sean de muy bajo porte en varios casos) en los ministerios vinculados a la producción y el trabajo (Cuadro 2). Sin embargo, existen aún varios ministerios de interés (como salud o turismo), entre otros organismos claves, donde no hay un tratamiento particular de la ESS, más allá de registrarse acciones o proyectos puntuales que no se 
han conformado como una política sostenida en el tiempo, que trascienda la buena voluntad de los elencos gubernamentales de turno. También destaca la falta de políticas a nivel de más intendencias, incluso en algunas con relevante peso de la ESS históricamente.

El alcance de las PPESS es principalmente nacional (80\%, 18 casos), mientras que las otras 5 responden a intendencias, que presentan solo una política de apoyo directo en la IC (desde 2015 también la IM cuenta con una Unidad de ESS).

A su vez, las que se concentran en el ámbito rural son 25\%, en el urbano $35 \%$ y las que operan en ambos $40 \%$ (Cuadro 1). Las rurales son todas de alto o medio vínculo con el fomento de la ESS, en la otra punta, las urbano-rurales es donde más pesan las de medio y bajo vínculo. A ello contribuye el que las políticas de las intendencias en general están abocadas al ámbito rural-urbano (80\%), siendo solo una de ellas específica del ámbito rural.

De los casos relevados, se definen 5 como proyectos, 6 como programas sectoriales, 4 intersectoriales y 5 como conjuntos de programas articulados y en menor medida se trata de políticas o planes de acción (3 casos) (Gráfico 1). ${ }^{7}$ No obstante, las políticas de vínculo alto con el apoyo a la ESS son mayoritariamente proyectos o programas sectoriales (75\%), es decir, de baja amplitud y/o cobertura.

Las políticas de apoyo directo a la ESS en su amplia mayoría cuentan con instrumento legal (Cuadro 2), y la mitad de ellas vía ley, lo que resulta positivo para su sostenibilidad.

Las principales áreas objetivo - para 22 políticas que pudieron priorizar-son:

Trabajo y empleo (10 casos).

Desarrollo económico local ( 7 casos en total si se le suma otras opciones vinculadas al desarrollo económico-productivo en general).

Educación y capacitación (2 casos).

Desarrollo rural, Bienestar social y Vivienda son priorizados por algún caso (cuadro 2).

En términos relativos, el peso del área objetivo Trabajo y empleo es mucho mayor en el primer gobierno del FA y en el segundo la de Desarrollo económico local, lo que podría estar acompañando el estado socioeconómico del país, marcado en esos años por bajos niveles de desempleo y cierta tendencia al crecimiento económico. Lo que también iría en línea con una visión general de una ESS como instrumento, más que como un objetivo en sí misma.

En concordancia con lo anterior, respecto al principal fin de la política, puestas a priorizar entre cuatro opciones: $48 \%$ priorizan la inclusión sociolaboral, 35\% aumentar la competitividad de los emprendimientos, y solo $9 \%$ en cada caso menciona las dos opciones pro ESS como tal (es decir, promover la ESS, sea porque se la considera una forma de hacer economía que también necesita apoyos o bien por lo deseable que son sus valores y características intrínsecas) (Cuadro 2). Incluso en casi todos los organismos específicos para la ESS, su búsqueda principal radica en la competitividad o bien la inclusión.

De una serie de áreas de acción sugeridas en la encuesta, existen cinco que sobresalen por ser realizadas por más del $60 \%$, no obstante, a la hora de priorizar según recursos asignados se destacan claramente dos: brindar crédito/financiamiento y acciones de asistencia técnica y organizativa. En un segundo plano aparecen formación y capacitación, apoyo a la comercialización y apoyo a la articulación de los emprendimientos en redes o cadenas (en este último caso, solo en políticas del MIEM y MGAP).

\section{C.2. Participación de actores en el proceso de la política}

En 17 casos (74\%) existen mecanismos de participación o de control social de la política.

Ello ocurre en todas las políticas previas a 2005 y solo mayoritariamente en las posteriores. Por institución, salvo en MIDES, MIEM, IM e IC donde solo 50\% aproximadamente presentan este tipo de mecanismos, en 
el resto los hay en todas las políticas. Se observa un alto grado de existencia de los mismos entre las políticas de vínculo alto y bajo con la ESS.

En los 6 casos en los que no existen mecanismos de participación/control de las políticas, se entiende por parte de sus referentes que debería existir, argumentando que ello es necesario para: la apropiación de la herramienta, dar continuidad a los procesos y que no dependa de la estabilidad de los convenios -y así devolver a la población lo trabajado y no irse cuando culminan los mismos-, para un necesario mayor compromiso de los emprendimientos y por razones democráticas y de control social.

Se destaca que, dentro de estos 6 casos, 3 son del MIDES (el 50\% de las políticas relevadas del ministerio), incluyendo políticas específicas para la ESS.

De entre aquellas 17 donde si hay este tipo de mecanismos, donde más se dan es en:

la propia ejecución de las acciones o actividades (12 casos de 17),

la planificación de las acciones o actividades (11 casos),

el monitoreo y evaluación de los resultados de la política ( 9 casos), y

la formulación de la política ( 8 casos).

Es mucho más bajo el número de políticas que cuentan con participación o control en materia de definición de los asociados - convenios- de la política y en el acompañamiento de la gestión financiera de la misma (4 casos respectivamente).

Ahora bien, cuando se consulta por el principal momento de la participación o control social, esto se da en la propia ejecución de las acciones o actividades (59\% de los 17 casos), o bien en la planificación de las mismas (23,5\%). Solo en un caso la participación es principal en el monitoreo y evaluación de los resultados y en ningún caso en las opciones restantes.

Respecto a los mecanismos existentes, en 4 de los 17 casos donde estos existen llega a darse participación o control en consejos específicos de dirección o gestión de la política. En 15 casos se da en grupos/mesas de trabajo compartidos con usuarios u otros participantes de la política, sean específicas de las políticas o más amplias como mesas sectoriales o departamentales. Finalmente, en 6 casos son consultas informales o reuniones específicas y no regulares de intercambio.

Los principales participantes en estos espacios/mecanismos de participación o control son claramente la representación de los propios emprendimientos beneficiarios (aunque en 5 de las 17 políticas no hay participación de este actor), lo que podría estar vinculado con la mayor participación/control en las etapas de planificación y ejecución de las políticas y no en las vinculadas a su concepción, forma de gestión y evaluación.

Luego le sigue la representación de otras agencias del gobierno y de las organizaciones/redes de articulación de los emprendimientos o de la ESS (aunque solo en 5 políticas hay participación de este último actor).

políticas hay participación de este último actor).

Las políticas directas de apoyo a la ESS, presentan mayor nivel relativo de participación de representantes de sus organizaciones/redes y sobre todo de los emprendimientos, que son casi exclusivamente los únicos actores que participan. Y con ello, como era de esperar, en estas políticas la participación/control se concentra solamente en la planificación y sobre todo en la ejecución de las actividades.

Si bien solo un caso de los 17 plantea que no son estables estos mecanismos, solo en 12 casos los mismos alcanzan niveles de estar establecidos por normativa, en el resto se trataría de mecanismos ad hoc o informales.

Respecto a la percepción sobre si la participación debería ser mayor o menor,

en las etapas donde existe mayor participación, vinculada a planificación y ejecución de tareas, todavía habría bastante para profundizar;

en las puntas, construcción de la política y sobre todo en su monitoreo y evaluación, prácticamente no priorizadas como principales fases donde se da actualmente la participación/control, se plantea que es necesario aumentarla; 
y en materia de gestión financiera y sobre todo de convenios de las políticas, no solo existe muy baja participación/control actual, sino que tampoco es muy alta la percepción sobre qué debería aumentarse.

Finalmente, también existieron algunos planteos respecto a las contrapartes de estos procesos, como la limitación de hecho existente por falta de organizaciones locales, que debe aumentarse la eficiencia de dicha participación dada la falta de tiempo disponible en las organizaciones para dedicarle al tema, o que el nivel de participación actual es el que éstas determinan, dado que no terminan de "apropiarse de la herramienta".

\section{C.3. Articulación externa}

\section{Articulación con otras políticas públicas}

El 52\% de las PPESS dice articular mucho con otras políticas públicas en general y otro 30\% articular "'relativamente", mientras que las restantes lo hacen poco, nadie menciona no articular. Respecto a articular específicamente con otras PPESS, 43,5\% y 26\% dicen hacerlo mucho y 'relativamente', mientras 30,5\% lo hacen poco o incluso nada. Es decir, que la articulación (mucha y más o menos) cae de $82 \%$ a $70 \%$ en ambas consultas.

Cuando se consulta sobre los mecanismos para la articulación con otras políticas destinadas hacia la ESS, los mismos aparecen como poco potentes y a veces no se refieren específicamente a ámbitos de apoyo directo a la ESS.

Ello podría ser parte de la explicación del problema planteado por muchos: existen emprendimientos sobreasistidos descoordinadamente, dándose problemas de incentivos poco claros y perjuicios para los emprendimientos y en la asignación de recursos públicos.

Posiblemente vinculado con ello, se tiene que el principal canal para identificar la demanda es vía presentación de los propios interesados (fundamentalmente mediante presentación de propuestas o proyectos -demanda abierta- o vía llamados públicos a proyectos), y luego muy lejos aparecen: por iniciativa del propio órgano responsable de la política; investigación, diagnóstico o encuesta para identificar demanda; y vía las organizaciones gremiales o de la sociedad civil en general. Ello en alguna medida podría estar indicando quizás cierta falta de planificación general de las políticas hacia la ESS (lo que podría sumarse con la poca articulación real entre ellas).

No obstante, las políticas de mayor vínculo con la ESS, que coordinan levemente menos que el resto con otras políticas en general, lo hacen algo más cuando se trata de PPESS.

Las PPESS de los gobiernos del FA plantean en su mayoría articular mucho o bastante, lo contrario ocurre cuando nos alejamos en el tiempo.

En MIDES y ministerios de la producción (MIEM y MGAP) hay diversidad de casos, en los otros ministerios más bien baja articulación. En OPP, intendencias e institutos públicos de derecho privado (INC e INCACOOP) se plantea alto nivel de articulación.

Dentro de las PPESS que articulan con otras PPESS, 85\% considera beneficiosa o muy beneficiosa dicha articulación, y solo $15 \%$ poco beneficiosa.

Formalizadas, aparece el $65 \%$ de tales articulaciones, en su gran mayoría vía acuerdos o convenios marco.

\section{Delegación de ejecución de politicas a terceros vía convenios y financiamiento}

Para la implementación directa de algunas áreas de acción existe 83\% de casos (19) que cuentan con convenios con otros actores (siendo en la mitad de ellos la principal forma de ejecución, al menos en algunas áreas de la política), en la mayoría de los casos con traspaso de fondos. Principalmente se tratan de otros órganos del gobierno nacional y/o municipal (en línea con el relativo alto porcentaje de articulación con 
otras políticas visto previamente) ${ }^{8}$ y con organizaciones de la sociedad civil.

Respecto a la PPESS de organismos del gobierno nacional, se destaca que solo en 2 casos se convenia/ articula con INACOOP. En cuanto a las pertenecientes a intendencias, solo se mencionan las propias intendencias y en general son con sus unidades de desarrollo.

Por su parte, menos de la mitad de las políticas que acuerdan con organizaciones de la sociedad civil lo hacen con organizaciones/redes de ESS, aunque de considerar también a entidades de apoyo a la ESS privadas, serían la mayoría.

Se destaca así que la mayoría de los casos presentan cierta delegación en la ejecución, en línea con la tendencia general a la tercerización de las políticas públicas, que, no obstante, dependiendo de su uso puede ser un instrumento de 'empoderamiento' de las entidades parte, significando un reflejo de políticas más participativas; tema no abordado en el mapeo.

\section{Bola de nieve}

Consultados los referentes de las PPESS sobre si conocen otras políticas públicas o programas que podrían ser considerados en este mapeo, a nivel nacional o municipal, alrededor de la mitad plantaron que sí. Luego, si bien esta consulta llega al final de la encuesta y muchos no le dieron la relevancia necesaria (lo que ciertamente en sí mismo es un indicador), y por tanto debe tomarse con cautela lo que sigue en este apartado, puede afirmarse que dentro de los pocos que pudieron efectivamente dar datos de otras políticas -ciertamente en general información no muy específica-, no muchas de las nombradas son parte de este mapeo, habiendo bastante desconocimiento al respecto.

Por ministerios se observa que en algunos de ellos (MIEM, MTSS, MVOTMA) no se nombra ninguna otra política, en otros como el MIDES, MGAP (sobre todo en este caso) o el INC se mencionan fundamentalmente políticas del propio ministerio o área particular (lo social o lo rural respectivamente). En las PPESS de intendencias, por su parte, solo se refiere o bien a políticas similares en otras intendencias o bien a los programas nacionales con los que tienen convenios. Finalmente, el INACOOP no resulta mencionado frecuentemente.

En las políticas más antiguas -de los noventa-, ninguna menciona otra política.

Finalmente, no hay aquí un patrón de respuesta controlando según nivel de vinculación con la ESS, mostrando igual desconocimiento incluso en aquellas de apoyo directo a la ESS.

\section{C.4. Posicionamiento conceptual}

Consultados los referentes de de las distintas políticas sobre desde qué perspectivas se posiciona cada una de ellas a la hora del trabajo con emprendimientos, 2 de las políticas menos vinculadas directamente con la ESS plantearon no saber en esta pregunta, al tiempo que lamentablemente no fueron consultadas las 2 correspondientes a la IC, una de las cuales es de las más importantes del país relacionadas al campo de la ESS y la otra seguramente la menos vinculada de las 23 .

De las 19 que contestaron:

la mayoría se paran desde el asociativismo (11) y/o desde el cooperativismo (8), mientras que 6 bajo la idea de emprendimientos sociales/comunitarios,

5 bajo la perspectiva de ESS y 2 de Economía Social.

En ninguno de los casos se parte explícitamente desde la concepción del tercer sector o desde la EcSol. Al tiempo que en 3 casos se plantean explícitamente perspectivas específicas: integración social y productiva, 
desarrollo territorial y cohesión social y MIPYMES. De todas formas, si se analizan las valoraciones y las acciones jerarquizadas por los entrevistados, parecen alinearse más hacia la expresión difusa de "tercer sector", enfatizando así las bondades del mismo para corregir las fallas del mercado y del Estado.

Respecto a cuál de estas perspectivas es la principal, nuevamente destacan el asociativismo (9) y el cooperativismo (4). Luego, 2 elijen la ESS, una la idea de emprendimientos sociales/comunitarios y, finalmente, las 3 que plantearon perspectivas específicas las ratifican como la principal.

No hay posicionamientos unánimes según entidades de pertenencia allí donde existe más de una, salvo en la IM donde las políticas relevadas, una de los noventa y otra más reciente, se paran desde la ESS; IM que desde 2015 a su vez cuenta con una Unidad de ESS.

Las PPESS de mayor vínculo (directas) con la ESS se posicionan todas desde el asociativismo o cooperativismo. En el otro extremo - pasando por la diversidad de situaciones de las de media vinculación-, las de bajo vínculo muestran como era esperable mayoría de otros marcos de posicionamiento o bien falta de definición al respecto.

En línea con lo anterior, se observa que el asociativismo es el público más difundido como destinatario de las 23 PPESS, si bien con escasa diferencia respecto a emprendimientos individuales y familiares, incluso a la hora de priorizar no existe predominancia.

A su vez, en su gran mayoría las PPESS trabajan con proyectos colectivos, siendo en el $40 \%$ de los casos el tipo exclusivo de proyectos a los que llega la política. Solo en dos casos aparentemente no se trabaja con este tipo de emprendimientos: microcrédito rural y la Unidad de Apoyo a MIPYMES de la IC (la política menos vinculadas a la ESS de las 23).

Las PPESS directas a la ESS, mayoritariamente se abocan a emprendimientos asociativos (75\%), mientras que solo una de vínculo medio lo hace y ninguna de vínculo bajo.

Respecto a la procedencia de los emprendimientos, están focalizados en ciertos sectores específicos según cada una de las políticas, donde lo rural y la condición de bajos ingresos son los recortes más extendidos.

\section{C.5. Evaluación y desafíos}

Las políticas han sido evaluadas en casi un 50\% de los casos de manera formal, mientras que en otro 35\% de manera informal ( 19 casos en total). Salvo un caso que no sabe, las otras 3 políticas (13\%) "no han sido evaluadas, pero se evaluarán en el futuro cercano".

Las políticas de alto vínculo con la ESS son relativamente de las menos evaluadas.

Existen indicadores de seguimiento o evaluación de resultado de la política en 15 casos (65\%), de los que la mitad son políticas con evaluación formal. En general se trata de aspectos cuantitativos, vinculados a número de emprendimientos o de población atendida, cantidad que ha establecido proyecto, se ha formalizado o ha reembolsado apoyos financieros, etc. En algunos casos se lo hace en términos comparados con la población total

que podría haber sido atendida o las zonas cubiertas, y en pocos casos se plantean variables de desempeño de los emprendimientos.

Consultados los referentes sobre cómo califican el cumplimiento de los objetivos previstos de las políticas en los últimos tres años, el 35\% (8 casos) expresa que se cumplieron y el otro 65\% plantea que se lo hizo parcialmente, no habiendo opiniones de no cumplimiento.

La amplia mayoría de políticas directas hacia la ESS expresan cumplimiento solo parcial.

En todos los casos se planteó que existen desafíos a futuro, y su identificación es de muy diversa índole. Se señala como el principal a los siguientes: mayor apoyo desde el gobierno nacional a la ESS (4 casos), mayores recursos o personal (3), necesidad de una transformación socio-cultural (3), y más participación y compromiso de las organizaciones sociales y los beneficiarios (3). 
Luego aparecen como el principal desafío -específicamente planteado fuera de las opciones de la pregunta-, en dos o tres ocasiones cada uno, la coordinación entre las políticas (buscando entre otras cosas no superponerse en campo) y la mejora técnica de los procesos productivos y/o productos de los emprendimientos. Un par de veces, también aparecen capacitación de los técnicos en la temática y la idea de poner en perspectiva al sector, considerando la economía en su conjunto y pensando en un posible programa para la ESS en el país. Finalmente, se mencionan una vez: la consolidación del proceso que viene llevando a cabo la política y, en otro caso, la ampliación de su cobertura geográfica.

De las 7 de 8 políticas de vinculación directa con la ESS que pudieron priorizar el principal desafío, 3 obedecen a cuestiones internas de la política, mientras el resto se trata o bien de la necesidad de transformación sociocultural o del desafío de mayor nivel de compromiso del gobierno nacional con un programa para la ESS.

En ningún caso de los 23 se menciona como el principal desafío la necesidad de una transformación en la matriz productiva.

En general, en este último sentido es mayor la visualización de la necesidad de un cambio sociocultural (12 casos) que productivo ( 6 casos), al tiempo que menos de la mitad (11 casos) plantean que es necesario un mayor apoyo desde el gobierno nacional a la ESS, si bien es la opción individual levemente más elegida antes de tener que priorizar la principal.

\section{D) Reflexiones finales}

Las dificultades varias para disponer de un panorama más detallado de los recursos que movilizan las diferentes políticas y los criterios de asignación y ejecución, no permiten un análisis profundo acerca de su significación en el conjunto del Estado. Lo mismo ocurre en materia de cobertura. La tendencia general observada es el carácter mayoritariamente subsidiario de las políticas hacia la ESS, y en algunos casos marginal, respecto a los rumbos generales de las políticas sectoriales e intersectoriales. De todas formas, el desafío es profundizar en lecturas transversales de los presupuestos públicos que permita dimensionarlas efectivamente, así como posibilitar su seguimiento y análisis continuo.

Así, en primer lugar, si bien aumentaron mucho las políticas de apoyo a la ESS desde 2005, inclusive hasta hoy, es necesario darles mayor densidad en cantidad, calidad y profundidad, tratando de reconocer mejor las especificidades y diversidad de la ESS en el país.

En efecto, se necesitan más políticas específicas para la ESS (en particular en intendencias y algunos ministerios, pero también en institutos públicos de derecho privado así como en agencias de educación e investigación, entre otros ámbitos), y que sean menos subsidiarias.

En segundo lugar, también es necesario alcanzar mucho mayor interinstitucionalidad entre las PPESS existentes. La creación de un espacio de articulación estable entre ellas quizás sería una primera medida, como por ejemplo una Coordinadora Nacional de PPESS. Ahora bien, también debe generarse trasversalidad con el resto de las políticas públicas, sobre todo -o al menos- en temáticas claves, como innovación, capacitación, desarrollo local, clusters, etc.

El INACOOP, que tiene dicha coordinación como principal desafío, quizás deba ser el principal promotor de tal interinstitucionalidad y transversalidad. Su visibilidad, como fue visto, no es muy elevada, y ampliarla es clave para el sector de la ESS y para estimular articulaciones horizontales y encadenamientos intersectoriales. Surge como gran desafío, entonces, la dinamización del INACOOP y su rol articulador en materias de políticas públicas hacia el sector, pluralizando a los sujetos participantes de las mismas (más allá del sector cooperativo) y propiciando e implementando ámbitos de coelaboración.

En tercer lugar, se requiere algún nivel de política de estado hacia la ESS si se quiere efectivamente un desarrollo significativo de la misma. La falta de visualización de otras esferas de la ESS más allá de los emprendimientos en sí mismos, la concentración en objetivos muy específicos, con escasa atención a 
los procesos de innovación y desarrollo tecnológico, o bien la baja importancia otorgada a la necesaria transformación de la matriz productiva y de los patrones de consumo y distribución para mejorar la suerte de la ESS, no se explican únicamente por lo pocas, pequeñas o específicas que son las PPESS mapeadas, dan cuenta de un proceso mayor que las contiene y les da forma.

A partir de esos desafíos generales, se propone jerarquizar tres núcleos de reflexiones interrelacionados que resultan claves para identificar, operacionalizar y profundizar a futuro los desafíos de las PPESS relevadas y, de alguna forma, de la ESS en general en el país.

\section{El desafío de profundizar la participación: integral e institucionalizada}

Más que el énfasis en lo vertical y jerárquico que implica la distinción entre políticas de arriba-abajo o abajo-arriba, las políticas para la economía social y solidaria conforman "un proceso horizontal y dinámico que implica a diversos actores sociales". Proceso que no puede ser lineal ni estático, y que requiere de un diálogo y aprendizaje continuo en los diversos niveles, desde lo nacional a lo local (Mendell, 2007, p. 57).

En la mayoría de las PPESS existen espacios/mecanismos de participación, a la vez que se la percibe como positiva y deseable, incluso se valora que debería ser mayor. Los modos cómo se construyen los valores que sustentan estas políticas son tan importantes como los acuerdos acerca de las acciones a priorizar y los contenidos a desarrollar por las mismas.

No obstante, la mayoría visualiza la participación en la ejecución de las políticas, pero no tanto en el diseño, planificación o evaluación de las mismas, y menos aún a la hora de seleccionar a los actores con que se convenía o en materia de la gestión financiera de la política. Lo cual condice con los espacios/mecanismos existentes, donde sólo en pocos casos llega a darse participación o control en consejos específicos de dirección o gestión de las políticas. Esto también se ve confirmado por quienes son sujetos de esa participación, siendo los principales participantes los propios beneficiarios. Así, por ejemplo, las redes o gremiales que nuclean a dichos beneficiarios podrían tener una visión más del conjunto, o al menos sectorial que pudiera ayudar en la planificación, gestión y evaluación de las PPESS.

Un desafío, entonces, está en constituir espacios nacionales, regionales y locales que permitan la coelaboración entre los diversos actores y escalas. Claro que este desafío no es solo para las PPESS, tal como algunas de ellas mencionan, sino que también se encuentran limitaciones desde los emprendimientos -que no terminan de entender que la construcción de vínculos con otros no es pérdida de tiempo, que juntarse con otros a pensar las PPESS así como la ESS en general es clave para su propia sustentabilidad y para la ampliación del sector-, o desde sus redes o federaciones -que no terminan de lograr ser verdaderas bisagras articuladoras entre la comunidad y el Estado, por una parte, y por el otra sus emprendimientos de base, pensando en construir un movimiento fuerte, amplio y dinámico-.

La participación directa de los involucrados podría ser una manera de hacer más efectivas las políticas públicas y que concretamente lleguen a quienes dicen atender. En este sentido, previendo que al delegar su ejecución a organizaciones de ESS la política pueda tener más penetración y atender a las necesidades concretas de los actores de ESS; habría que ver si las redes o federaciones podrán hacer frente a este desafío en forma compartida con políticas territoriales y territorializadas. Para que la participación no sea meramente en la ejecución, lo planteado anteriormente, respecto a los foros nacionales, regionales y locales de economía solidaria resultan claves, siempre que movilicen recursos materiales efectivos y criterios compartidos, orientados crecientemente a la ampliación y defensa de lo común.

Por último, un aspecto a destacar en este sentido es que si bien la mayoría de los casos plantea que los espacios/mecanismos de participación son estables, estos no llegan a estar establecidos por normativa. Esto deja la participación en la PPESS supeditada a las intencionalidades de quienes las ejecutan, pero no siendo parte intrínseca de su realización. 


\section{El desafío de una mayor articulación: interinstitucional y transversal}

La mayoría de las PPESS encuestadas dicen articular mucho o bastante y que ello es beneficioso, pero al desagregar esa articulación en acciones concretas nos encontramos con mecanismos poco potentes, que no parecen hacer dicha articulación realmente efectiva. Pueden entenderse estas respuestas en el marco de la creciente percepción positiva sobre la articulación, un "deber ser" en cuanto a cómo se percibe la mejor manera de hacer política pública, pero que en los hechos no se traduce de manera fuerte en su accionar.

En efecto, como se pudo visualizar, de existir articulación la misma se da al interior del organismo de pertenencia y raramente de manera interinstitucional. Este aspecto se da menos entre las intendencias encuestadas, que se conciben a sí mismas como espacios de articulación, pero se percibe fuertemente en la PPESS dependientes de ministerios.

Un punto que ya fue destacado como desafío es la poca articulación que se menciona con el INACOOP, y siendo que este es el único organismo nacional que tiene por objetivo directo al cooperativismo, se convierte en un actor clave en la articulación de las PPESS para aunar esfuerzo hacia el sector y potenciar su efectividad. En este sentido, el INACOOP debería pensar en qué forma incluye los nuevos actores del campo de la ESS en el país. Potenciar su rol articulador entre políticas y promover la vinculación entre las políticas y los demandantes de la misma es un punto medular, dado que casi todas las PPESS dicen surgir de la demanda e implican la presentación de proyectos para acceder a ellas.

La interinstitucionalidad y la importancia de los espacios de concertación de las políticas, mencionados antes en relación a la participación, interpelan al conjunto de la institucionalidad pública a los efectos de evitar la dispersión, superposición en cuanto a los objetivos y la generación de compartimentos estancos entre las mismas.

Una mirada de conjunto, como genera este mapeo, permite dimensionar la fragmentación de las políticas y programas, muchas entidades haciendo pocas cosas, articulando poco entre sí y, sobre todo, partiendo de diversas concepciones de hacia dónde ir.

Los espacios de concertación son necesarios para incrementar y habilitar la articulación. Hacer consciente el debate acerca de hacia dónde se quiere ir, para construir una visión articulada y consensuada sobre cómo se define el campo de la ESS y qué se quiere lograr con la toma de posición del Estado respecto a la misma. Pareciera necesario reafirmar el carácter de políticas públicas como campo siempre en disputa y no reducido al gobierno y las estructuras institucionales. Surge como desafío la necesidad de pensar conjuntamente el para qué, más allá de diseñar múltiples y pequeñas intervenciones estatales. Debe procurarse penetrar en el conjunto de las políticas sociales, culturales y productivas del país.

Más allá de lo dicho, el análisis de las PPESS identificadas, muestra la existencia de un conjunto de políticas que se han ido sosteniendo en el tiempo y donde el apelo a lo asociativo ha crecido en los últimos lustros. Es notorio, en este sentido, el desconocimiento existente entre los propios organismos acerca de los planes, programas y proyectos orientados a la ESS que se desarrollan en el país; siendo un desafío clave socializar la información y mejorar las estrategias de comunicación de las políticas.

Un aspecto relevante que surge del mapeo general realizado es el papel, centralidad y visibilidad pública dentro de las políticas que tiene el cooperativismo, siendo la EcSol o en general la ESS expresiones con menor reconocimiento. Resulta clave que las cooperativas asuman mayor responsabilidad respecto a la ESS, capitalizando lo que por su trayectoria se ha ido ganando, a los efectos de ampliar el espacio público e incluir nuevas formas asociativas orientadas a la transformación social en sus demandas.

En este sentido, el desafío de la articulación de los propios actores centrales de la ESS implica debates profundos dentro de las entidades del sector, no exentos de tensiones, puesto que interpelan al cooperativismo que no se limita a constituirse como instrumento de resolución de necesidades, sino que trabaja por la construcción de nuevos modos de vida con vocación transformadora de la sociedad. También interpelan a la propia Coordinadora de Economía Solidaria y su estrategia de profundización de alianzas 
y espacios, exigiendo ampliar la articulación con las organizaciones y redes que surgen y se consolidan en espacios territoriales diversos, como son entre otras la Red de Semillas, Red de Agroecología, asociaciones de artesanos, empresas recuperadas, permacultura.

La demanda acerca de una mayor articulación de las políticas va acompañada entonces de la necesidad de potenciar los espacios de articulación entre los actores, para que las visiones restringidas respecto al accionar de cada una de las entidades concebidas aisladamente de paso a la confluencia hacia una visión sistémica e integrada, fuertemente enraizada en las localidades, las regiones, para luego pasar al nivel nacional e internacional.

\section{El desafío estratégico-conceptual de para qué: una ESS sistémica para el cambio social}

El posicionamiento de las PPESS parece corresponderse con una perspectiva de fondo cercana a la idea de tercer sector o a la visión europea de Economía Social, visualizando a este campo como un sector que puede resultar beneficioso impulsar por sus capacidades como paliativo de las "deficiencias" del mercado y del propio estado. Así es que sus objetivos centrales se enmarcan en la inclusión sociolaboral coincidiendo con sus áreas de acción signadas por la competitividad de los emprendimientos como preocupación principal (financiamiento, asistencia técnica, etc.).

Se puede visualizar así, en las valoraciones de los organismos, cierta idea de que se trata de un juego a tres bandas: sector público estatal, sector privado empresarial y el sector asociativo y solidario con el consiguiente riesgo de no visualizar las interrelaciones, contradicciones y conflictos entre esos ámbitos. La idea de ir construyendo un sistema alternativo, otra forma de hacer economía y sociedad, no parece estar en el horizonte de estas políticas analizadas.

$\mathrm{Al}$ respecto, 25\% de las PPESS que tienen como fundamento a la ESS procuran como fin principal su fomento en sí, el resto prioriza o la competitividad de los emprendimientos (25\%) o la inclusión sociolaboral (50\%); parece necesario ir más allá de la generación de empleo y de la competitividad como horizonte.

Resulta necesario jerarquizar en la propia formulación y diseño de las políticas y en su conceptualización y concepción, un horizonte común del fomento de la ESS que posibilite construir relaciones y encadenamientos entre las políticas, por lo que la discusión sobre las finalidades, los para qué y con quiénes resulta clave.

Es fundamental entonces, desde el Estado, superar la visión residual de las PPESS de pobres para pobres o en el mejor de los casos de aporte socioeconómico marginal al desarrollo del país, consolidando un sistema de fomento y protección del trabajo asociativo, cooperativo y de ESS fuertemente articulado con el desarrollo de la industria nacional a diferentes escalas, propiciando encadenamientos productivos y redes territoriales y comunicacionales. Ello debe ser parte de la ampliación de los horizontes de tales políticas y materia presente en el conjunto de sus políticas.

Además, desde la ESS disputar otra concepción acerca de las políticas, que apunte a la construcción de políticas genuinas en estas áreas, bajo una racionalidad acorde con los principios de la ESS, con una fuerte, clara y amplia participación de los implicados.

Si bien requeriría por supuesto un análisis en profundidad al menos similar al hecho aquí a 2015, estando cerca del cierre del tercer gobierno progresista 2015-19, se entiende que el mismo ha sido de consolidación y continuidad de lo actuado previamente en PPESS.

En efecto, se han consolidado ciertas políticas de las aquí relevadas, ordenado o racionalizado otras, al tiempo que se han desarrollado en colaboración entre organismos públicos y privados nuevos instrumentos (una incubadora de cooperativas de base tecnológica -INCUBACOOP-, un programa de amplio alcance para capacitación y consultoría a emprendimientos -PROCOOP-, se ha avanzado de cara a la puesta en marcha de ciertos programas de compras públicas hacia la ESS, se discute un plan nacional de agroecología, 
entre otras acciones); pero seguramente las reflexiones aquí planteadas, su núcleo duro, no ha tenido cambios significativos.

En este sentido, la evaluación de para qué las políticas públicas fomentan lo asociativo o la ESS en general, y en particular en los últimos lustros donde más han aumentado en términos históricos, siempre es una pregunta pertinente y sigue siendo un debate abierto.

Si de cara a la trasformación social es imprescindible "democratizar el estado" y reducir la "tiranía del mercado", contar con un sector-movimiento democrático y solidario de la sociedad fuerte y con capacidad de desarrollar 'crecientemente otra economía', podría -sino debería- ser una búsqueda clave de un gobierno progresista.

\section{Bibliografía Citada}

Chaves, R. (2012). Las políticas públicas y las cooperativas. Ekonomiaz, 79.

Confederación Uruguaya de Entidades Cooperativas (2014). Propuestas programáticas del cooperativismo uruguayo a los partidos politicos. Montevideo: CUDECOOP.

Coraggio, J. L. (2014). La presencia de la ESS y su institucionalización en América Latina. Documento Ocasional 7 , de la serie: Potencial y Límites de la ESS. Instituto de Investigación de Naciones Unidas para el Desarrollo Social (UNRISD).

Hintze, S. (2010). La politica es un arma cargada de futuro: La economía social y solidaria en Brasily Venezuela. Buenos Aires: CLACSO.

Martí, J. P., Soria, C. y Dabezies, M. J. (2008). Programas públicos para el fomento del cooperativismo de trabajo en Uruguay (1976-2006). En La Economía Social en Iberoamérica. Enfoques de interés en España, Portugal y Uruguay. Montevideo: Divina Pastora-CUDECOOP-Fundibes.

Mendell, M. (2007). La co-producción de políticas sociales en Québec: El caso de la Economía Social. En Vuotto, M. (coordinadora), La co-construcción depolíticas públicas en el campo de la economía social. Buenos Aires: Prometeo.

Méndez, V. y Romero, M. (2012). Economía Social y Solidaria. Concepto y Políticas Públicas en Uruguay. En Vulnerabilidad y exclusión. Aportes para las politicas sociales. Uruguay Social (Vol. 5). Montevideo: MIDES-FCSUdelaR.

Torrelli, M. (2013). El sector cooperativo y de la ESS visto a través de algunas de sus principales variables (Capítulo 4 y 5). Montevideo: OMERCOOPES (PROCOOPSUR, RECM) - AECID. Mimeo.

\section{Notas}

1 Proyecto llevado adelante entre 2013 y 2015 desde la Unidad de Estudios Cooperativos del Servicio Central de Extensión y Actividades en el Medio de la Unidad de la República (UEC-SCEAM-UdelaR)2004-2017) y co-financiado por la comisión Sectorial de Investigación Cientifica (CSIC-UDELAR) y el Instituto Nacional del Cooperativismo (INACOOP). En su implantación participó un amplio equipo de trabajo a quien también le corresponden los resultados de este artículo, que surge en gran medida del Informe General del proyecto, que junto a otros materiales vinculados al mismo, puede verse en: http://mapeoecsol.blogspot.com/

2 Se los incluyó no solo por ser buena parte de las redes comprendidas en la ESS nacional, sino porque se considera teóricamente que lo que es o se procura que sea solidario es el circuito económico, situándose en el centro a las personas y actuando necesariamente junto a otros en alguna de las fases de la actividad económica (producción, consumo, distribución y finanzas). Según el lema de la EcSol, "se puede ser pequeño, pero nunca aislado", quedando así de manifiesto la centralidad de las 'redes' en la EcSol. Así, en el mapeo se exigió que estos emprendimientos (individuales o familiares) se vinculen voluntariamente a una red de EcSol. Por una discusión más amplia del punto, ver el ya citado Informe General del proyecto mapeo.

3 Un mapeo no es un censo, no se tiene previamente el universo a relevar, se lo va construyendo. 
4 Se dejó fuera del mapeo a los institutos de educación pública.

5 Por ejemplo, el INACOOP realiza distintos programas, no obstante, se lo entendió una sola política con unidad. En el caso de ministerios o intendencias con varios proyectos y programas, el criterio sobre puntos de corte y diferenciación fue definido junto con sus referentes.

$6 \mathrm{Al}$ respecto vale señalar que los propios beneficiarios co-financian parte de la política en cinco casos (22\% de los 23 casos totales): tres políticas del MIEM, una del MVOTMA y otra del INACOOP. En los primeros cuatro casos se trata de complementos o contraprestaciones que exige la propia política (aportan entre 5\% y $15 \%$ del costo total de las políticas), y en el último de la prestación coactiva que todas las cooperativas deben abonar al financiamiento del mismo ( $44 \%$ del financiamiento total del INACOOP al momento del mapeo).

7 Los proyectos constituyen la unidad mínima de acción para el logro de uno o más objetivos, al tiempo que los programas se conforman por un conjunto de proyectos, sobre los cuales establecen las prioridades de intervención, definen el marco institucional y asignan los recursos. Las políticas o planes de acción, alcanzan un nivel aún más general de cobertura, siendo directrices generales para la acción de proyectos o programas.

8 En igual dirección vale mencionar que la segunda fuente de financiamiento en importancia (luego de fondos públicos propios o del presupuesto nacional, mayoritaria en 22 de los 23 casos) es la proveniente de otros organismos del estado, lo que se da en 8 casos (35\% de las 23). 